Vietnam Journal of Mechanics, VAST, Vol.40, No. 2 (2018), pp. 155-162

DOI:10.15625/0866-7136/10732

\title{
WEIGHTED AVERAGING TECHNIQUE FOR THE DESIGN OF DYNAMIC VIBRATION ABSORBER INSTALLED IN DAMPED PRIMARY STRUCTURES
}

\author{
N. D. Anh $^{1}$, N. Q. Hai ${ }^{2}$, N. X. Nguyen ${ }^{3, *}$, D. V. Hieu ${ }^{4}$, P. T. T. My $^{1}$ \\ ${ }^{1}$ Institute of Mechanics, VAST, Hanoi, Vietnam \\ ${ }^{2}$ Hanoi Architechtural University, Vietnam \\ ${ }^{3}$ VNU University of Science, Hanoi, Vietnam \\ ${ }^{4}$ Thai Nguyen University of Technology, Vietnam \\ *E-mail: nguyennx12@gmail.com \\ Received September 20, 2017
}

\begin{abstract}
The dynamic vibration absorber (DVA) has been widely applied in various technical fields. This paper presents a simple approach to determine a closed-form expression for the tuning ratio of a DVA attached to a damped primary structure. The result is obtained by using the so-called weighted averaging technique of the equivalent linearization method proposed by the first author. The values of the tuning ratio given in this paper are compared with those obtained numerically as well as the ones obtained from other authors. The comparison shows the reliability of the method given in this study.

Keywords: Dynamic vibration absorber, tuned mass damper, analytical formula, damped structures.
\end{abstract}

\section{INTRODUCTION}

The dynamic vibration absorber (DVA) is a passive control system installed in a primary structure to suppress harmful vibration. Frahm [1] introduced the first DVA and then Ormondroyd and Den Hartog [2] proposed the standard model of DVA with a spring element and a viscous element are arranged in parallel. Since then, many studies on DVA have been investigated [3-5].

In order to maximize the effect of DVA on the primary structure, two main design parameters of a DVA are the tuning ratio and damping ratio. Den Hartog [6] presented two simple expressions for the optimal tuning ratio and damping ratio of a DVA by minimizing the displacement response of an undamped primary structure subjected to sinusoidal excitation. When the primary structure taking into account damping, there have been either numerical solutions or empirical expressions for optimal parameters of DVA in the literature [7-11].

(C) 2018 Vietnam Academy of Science and Technology 
In 2007, Ghosh and Basu [12] gave a closed-form expression for optimal tuning ratio of DVA by using an approximate assumption about the existence of two fixed points. Later, Anh and Nguyen [13] proposed another closed-form expression for the optimal tuning ratio of DVA. That result was obtained via the equivalent linearization method, where the original damped structure was replaced equivalently by an undamped structure and then using the known result for undamped structures to give the expression of optimal tuning ratio.

In this paper, the authors use a technique of the equivalent linearization method with a weighted averaging to replace the damped system by an undamped structure, and then using the known result for undamped structures given by Den Hartog to get the expression of optimal tuning ratio. The result in this study is compared with the results obtained from Ghosh and Basu's expression, Anh and Nguyen's expression as well as the result obtained numerically from Ioi and Ikeda [7]. The comparisons show that the values of optimal tuning ratio derived from the expression in this paper are closer to the values from the result given by Ioi and Ikeda than those from the expression proposed by Ghosh and Basu as well as by Anh and Nguyen.

\section{DEN HARTOG'S CLASSICAL RESULTS IN THE CASE OF UNDAMPED STRUCTURE}

A system consisting of a dynamic vibration installed in the primary structure is shown in Fig. 1. The primary structure includes a main mass $m_{s}$, a spring element $k_{s}$ and a damping element $c_{s}$ and is subjected an external force $f(t)$. The mass of the DVA is $m_{d}$ and its spring and damping coefficients are $k_{d}$ and $c_{d}$.

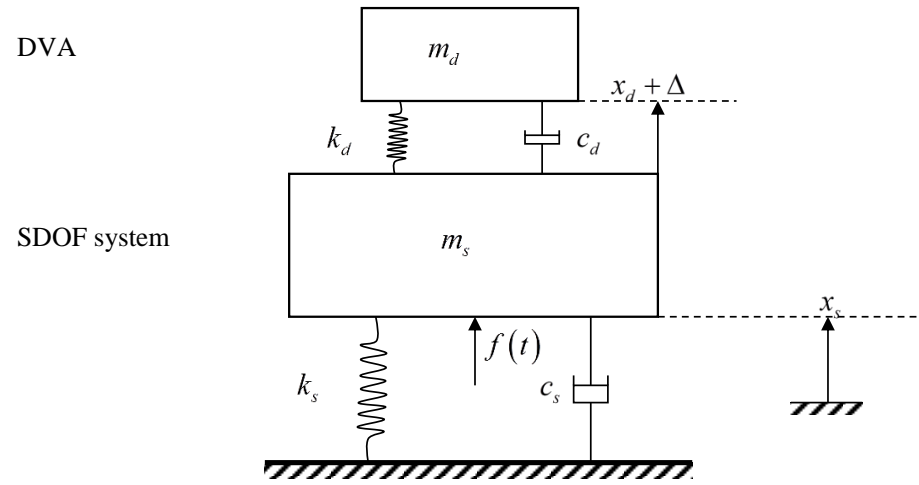

Fig. 1. Damped vibration absorber applied to a force-excited system with damping

Let $x_{s}$ and $x_{d}$ denote the displacements of the primary structure and the DVA, respectively. By using Lagrange's equations, we get the equations of motion

$$
\begin{aligned}
& m_{s} \ddot{x}_{s}+c_{s} \dot{x}_{s}+k_{s} x_{s}=c_{d} \dot{x}_{d}+k_{d} x_{d}+f(t), \\
& m_{d} \ddot{x}_{d}+c_{d} \dot{x}_{d}+k_{d} x_{d}=-m_{d} \ddot{x}_{s} .
\end{aligned}
$$


We introduce the dimensionless parameters as follows

$$
\begin{aligned}
& \mu=\frac{m_{d}}{m_{s}}, \quad \omega_{s}=\sqrt{\frac{k_{s}}{m_{s}}}, \quad \xi_{s}=\frac{c_{s}}{2 m_{s} \omega_{s}}, \\
& \omega_{d}=\sqrt{\frac{k_{d}}{m_{d}}}, \quad \xi_{d}=\frac{c_{d}}{2 m_{d} \omega_{d}}, \quad \alpha=\frac{\omega_{d}}{\omega_{s}},
\end{aligned}
$$

where $\mu$ is the mass, $\omega_{s}, \xi_{s}$ and $\omega_{d}, \xi_{d}$ are natural frequencies and damping ratios of the primary structure and of the DVA, respectively, $\alpha$ is the tuning ratio.

Eq. (1) can be rewritten as

$$
\begin{gathered}
\ddot{x}_{s}+2 \omega_{s} \xi_{s} \dot{x}_{s}+\omega_{s}^{2} x_{s}=2 \mu \alpha \xi_{d} \omega_{s} \dot{x}_{d}+\mu \alpha^{2} \omega_{s}^{2} x_{d}+\frac{f(t)}{m_{s}}, \\
\ddot{x}_{d}+2 \alpha \xi_{d} \omega_{s} \dot{x}_{d}+\alpha^{2} \omega_{s}^{2} x_{d}=-\ddot{x}_{s} .
\end{gathered}
$$

Den Hartog [6] presented the expressions for the optimal tuning ratio and damping ratio of a DVA in the case of undamped primary structures as follows

$$
\begin{aligned}
& \alpha_{\text {opt }}=\frac{1}{1+\mu}, \\
& \xi_{\text {dopt }}=\sqrt{\frac{3 \mu}{8(1+\mu)}} .
\end{aligned}
$$

In next sections, the authors give an approximate expression for the tuning ratio of a DVA installed in damped primary structures.

\section{USING EQUIVALENT LINEARIZATION METHOD WITH A WEIGHTED AVERAGING TO OBTAIN A CLOSED-FORM EXPRESSION FOR THE OPTIMAL TUNING RATIO}

The main purpose in this work is using the equivalent linearization method with a weighted averaging in order to replace approximately the original damped structure by an undamped structure and then using Den Hartog's results (5) to obtain the closed-form expression for optimal tuning ratio.

\subsection{The equivalent linearization method}

In Fig. 2(a) with damped structure, the equation of motion is

$$
\ddot{x}_{s}+2 \xi_{s} \omega_{s} \dot{x}_{s}+\omega_{s}^{2} x_{s}=0 .
$$

In Fig. 2(b) with undamped structure, the equation of motion has the form as follows

$$
\ddot{x}_{s}+\omega_{e}^{2} x_{s}=0,
$$

where $\omega_{e}$ is an unknown constant and will be determined by the following criterion

$$
A=\left\langle\left(2 \xi_{s} \omega_{s} \dot{x}_{s}+\omega_{s}^{2} x_{s}-\omega_{e}^{2} x_{s}\right)^{2}\right\rangle \rightarrow \underset{\omega_{e}}{\operatorname{Min}}
$$




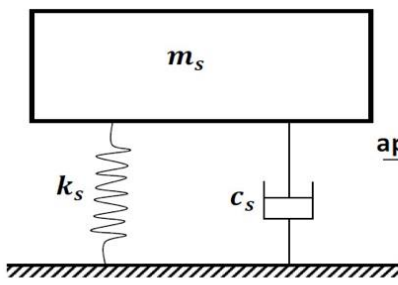

(a)

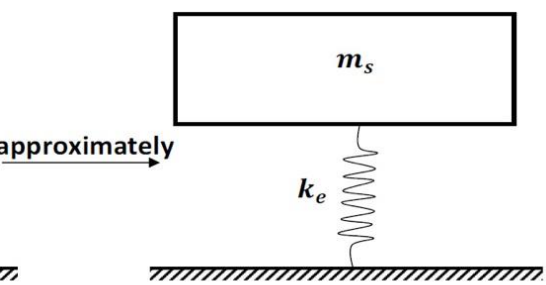

(b)

Fig. 2. The approximation of the primary structure

with

$$
\langle\cdot\rangle=\frac{1}{T} \int_{0}^{T}(\cdot) d t .
$$

Using Eq. (8), we get

$$
\frac{\partial A}{\partial \omega_{e}}=0
$$

This deduces

$$
\left(\omega_{e}^{2}-\omega_{s}^{2}\right)\left\langle x_{s}^{2}\right\rangle-2 \xi_{s} \omega_{s}\left\langle\dot{x}_{s} x_{s}\right\rangle=0
$$

Instead of using the averaging in Eq. (9), we define the weighted averaging in the next section.

\subsection{A weighted averaging}

In this subsection the weighted averaging technique proposed in [14] and has been applied to analyze some vibrating systems with nonlinearities [15] is shortly introduced. In practice, for a given dataset, the most common statistic is the arithmetic mean. The concept of average of a data set can be extended to functions. The conventional average value $(\mathrm{CAV})$ of an integrable deterministic function $x(t)$ on a domain $D:(0, d)$ is a constant value defined by

$$
\langle x(t)\rangle=\frac{1}{d} \int_{0}^{d} x(t) d t
$$

In many cases when the function $x(\omega t)$ is periodic with period $2 \pi / \omega$ the value $d$ is taken as $2 \pi / \omega$ and it leads to the averaged value of $x(t)$ over one period

$$
\left\langle\langle x(\omega t)\rangle=\frac{\omega}{2 \pi} \int_{0}^{2 \pi / \omega} x(\omega t) d t=\frac{1}{2 \pi} \int_{0}^{2 \pi} x(\tau) d \tau,\right.
$$

where $\tau=\omega t$ is the new variable or "new time". Averaged values play surely major roles in the past and at the present, however, the definition (11) has some deficiencies, for example, if Eqs. (11) or (12) are equal zero, the information about $x(t)$ is lost. For all harmonic functions $\cos (n \omega t)$ and $\sin (n \omega t)$, this observation is true. The dual approach to averaged values may be a possible way to suggest an alternative choice for the conventional average value, namely the constant coefficient $1 / d$ in (11) can be extended to a weighted coefficient as a function $h(t)$. Thus one gets the so-called a weighted average value 


$$
W(x(t))=\int_{0}^{d} h(t) x(t) d t,
$$

where the condition of normalization is satisfied as

$$
\int_{0}^{d} h(t) d t=1
$$

There are three basic weighted coefficients as follows:

- Basic optimistic weighted coefficients: They are increasing functions of $t$ and denoted as $O(t)$. Examples are $\alpha t^{\beta}$ and $\alpha e^{\beta t}, \alpha, \beta>0$.

- Basic pessimistic weighted coefficients: They are decreasing functions of $t$ and denoted as $P(t)$. Examples are $\alpha t^{\beta}$ and $\alpha e^{\beta t}, \alpha<0, \beta>0$; or $\alpha>0, \beta<0$.

- Neutral weighted coefficients: They are denoted as $N(t)$, where $N(t)$ is a constant.

An arbitrary weighted coefficient $h(t)$ can be obtained as summation and/or product of basic weighted coefficients. An example is

$$
h(t)=\sum_{i=1}^{n} A_{i} O_{i}(t)+B_{i} P_{i}(t)+C_{i} O_{i}(t) P_{i}(t)+N(t),
$$

where $A_{i}, B_{i}, C_{i}$ are constant.

In this paper, we will consider only $\omega$-periodic functions $x(\omega t)$. A special form of weighted coefficient is introduced as

$$
h(t)=s^{2} \omega^{2} t e^{-s \omega t}, \quad s>0 .
$$

\subsection{The closed-form expression of optimal tuning ratio}

Eq. (7) has a periodic solution as follows

$$
x_{s}=a \cos \omega_{e} t \text {, }
$$

with the weighted coefficient is

$$
h(t)=s^{2} \omega_{e}^{2} t e^{-s \omega_{e} t}, \quad s>0 .
$$

We will determine

$$
\begin{aligned}
W\left(x_{s}^{2}\right) & =W\left(a^{2} \cos ^{2} \omega_{e} t\right)=\int_{0}^{+\infty} a^{2} s^{2} \omega_{e}^{2} t e^{-s \omega_{e} t} \cos ^{2}\left(\omega_{e} t\right) d t \\
& =a^{2} \frac{s^{4}+2 s^{2}+8}{s^{4}+8 s^{2}+16} \\
W\left(\dot{x}_{s} x_{s}\right) & =W\left(-a^{2} \omega_{e} \sin \left(\omega_{e} t\right) \cos \left(\omega_{e} t\right)\right) \\
& =-\frac{a^{2} \omega_{e}}{2} \int_{0}^{+\infty} s^{2} \omega_{e}^{2} t e^{-s \omega_{e} t} \sin \left(2 \omega_{e} t\right) d t \\
& =-\frac{2 \omega_{e} a^{2} s^{3}}{s^{4}+8 s^{2}+16} .
\end{aligned}
$$


Substituting Eqs. (18) and (19) into Eq. (10) we obtain

$$
\left(s^{4}+2 s^{2}+8\right) \omega_{e}^{2}+4 s^{3} \omega_{s} \xi_{s} \omega_{e}-\left(s^{4}+2 s^{2}+8\right) \omega_{s}^{2}=0 .
$$

Eq. (20) leads to

$$
\omega_{e}=\frac{-2 s^{3} \xi_{s}+\sqrt{4 s^{6} \xi_{s}^{2}+\left(s^{4}+2 s^{2}+8\right)^{2}}}{s^{4}+2 s^{2}+8} \omega_{s} .
$$

Using Den Hartog's result (5) for undamped primary structure, we have

$$
\alpha_{\text {eopt }}=\frac{1}{1+\mu} \text {. }
$$

Noting that

$$
\alpha_{\text {eopt }}=\frac{\omega_{d}}{\omega_{e}}, \quad \alpha_{o p t}=\frac{\omega_{d}}{\omega_{s}},
$$

and using Eq. (21), finally we obtain a closed-form expression for the optimal tuning ratio as follows

$$
\alpha_{o p t}=\frac{-2 s^{3} \xi_{s}+\sqrt{4 s^{6} \xi_{s}^{2}+\left(s^{4}+2 s^{2}+8\right)^{2}}}{(1+\mu)\left(s^{4}+2 s^{2}+8\right)} .
$$

The expression (22) will reduce to Den Hartog's result (5) in the case of undamped primary structures. The formula (22) is independent of the damping ratio of the DVA. The optimal turning ratio in Eq. (22) depends not only on $\mu$ and $\xi_{s}$ but also on a parameter $s$. In this paper, for sakes of computation convenience, the chosen parameter $s$ is equal to 2 .

\section{COMPARISONS}

The values of the optimum tuning ratio from the proposed expression (22) will be compared with the values calculated from the empirical expression given by Ioi and Ikeda [7] as well as the expressions proposed by Ghosh and Basu [12] and by Anh and Nguyen [13]. In Ghosh and Basu's paper, they have given an expression

$$
\alpha_{o p t}=\sqrt{\frac{1-4 \xi_{s}^{2}-\mu\left(2 \xi_{s}^{2}-1\right)}{(1+\mu)^{3}}} .
$$

Meanwhile, Anh and Nguyen's expression is

$$
\alpha_{o p t}=\frac{1}{(1+\mu)\left(\sqrt{1+\frac{4}{\pi^{2}} \xi_{s}^{2}}+\frac{2}{\pi} \xi_{s}\right)} .
$$

The comparison is done in Tabs. 1 and 2, in which the mass ratio $\mu$ is taken to be 0.03 and 0.05 , respectively, and the different values of the structural damping ratio $\xi_{s}$ vary from 0.005 to 0.15 .

It is shown from Tabs. 1 and 2 that the values of optimal turning ratio from the expression (22) proposed in this paper are closer to the values from the empirical expression given by Ioi and Ikeda than those derived from the expression (23) given by Ghosh and Basu as well as from the expression (24) given by Anh and Nguyen. It also can be seen from Tabs. 1 and 2 that the values of turning ratio decrease when the structural ratio or the mass ratio increases. 
Table 1. Optimal turning ratio of TMD for different structural damping ratios and the mass ratio $\mu=0.03$

\begin{tabular}{lllll}
\hline$\xi_{s}$ & $\begin{array}{l}\alpha_{o p t} \\
\text { given by Ioi } \\
\text { and Ikada }\end{array}$ & $\begin{array}{l}\alpha_{\text {opt }} \\
\text { given by Ghosh } \\
\text { and Basu }\end{array}$ & $\begin{array}{l}\alpha_{o p t} \\
\text { given by Anh } \\
\text { and Nguyen }\end{array}$ & $\begin{array}{l}\alpha_{o p t} \\
\text { in present } \\
\text { the paper }\end{array}$ \\
\hline 0.005 & 0.9694 & $0.9708(0.14)^{*}$ & $0.9678(0.17)$ & $0.9684(0.10)$ \\
0.01 & 0.9679 & $0.9707(0.29)$ & $0.9647(0.33)$ & $0.9660(0.19)$ \\
0.02 & 0.9647 & $0.9701(0.56)$ & $0.9586(0.63)$ & $0.9612(0.36)$ \\
0.03 & 0.9613 & $0.9692(0.82)$ & $0.9525(0.92)$ & $0.9564(0.51)$ \\
0.05 & 0.9540 & $0.9661(1.27)$ & $0.9405(1.42)$ & $0.9469(0.74)$ \\
0.07 & 0.9464 & $0.9615(1.64)$ & $0.9286(1.84)$ & $0.9375(0.89)$ \\
0.1 & 0.9325 & $0.9515(2.04)$ & $0.9110(2.31)$ & $9.9235(0.96)$ \\
0.12 & 0.9225 & $0.9429(2.17)$ & $0.8995(2.49)$ & $0.9144(0.88)$ \\
0.14 & 0.9118 & $0.9326(2.28)$ & $0.8882(2.59)$ & $0.9053(0.71)$ \\
0.15 & 0.9062 & $0.9268(2.27)$ & $0.8826(2.60)$ & $0.9008(0.59)$ \\
\hline
\end{tabular}

${ }^{*}$ Different from Ioi and Ikeda's results in percentage terms

Table 2. Optimal turning ratio of TMD for different structural damping ratios and the mass ratio $\mu=0.05$

\begin{tabular}{lllll}
\hline$\xi_{s}$ & $\begin{array}{l}\alpha_{o p t} \\
\text { given by Ioi } \\
\text { and Ikeda }\end{array}$ & $\begin{array}{l}\alpha_{\text {opt }} \\
\text { given by Ghosh } \\
\text { and Basu }\end{array}$ & $\begin{array}{l}\alpha_{o p t} \\
\text { given by Anh } \\
\text { and Nguyen }\end{array}$ & $\begin{array}{l}\alpha_{o p t} \\
\text { in present } \\
\text { the paper }\end{array}$ \\
\hline 0.005 & 0.9508 & $0.9523(0.16)$ & $0.9494(0.15)$ & $0.9500(0.08)$ \\
0.01 & 0.9491 & $0.9522(0.33)$ & $0.9463(0.30)$ & $0.9476(0.15)$ \\
0.02 & 0.9456 & $0.9516(0.63)$ & $0.9403(0.56)$ & $0.9429(0.29)$ \\
0.03 & 0.9420 & $0.9507(0.92)$ & $0.9344(0.81)$ & $0.9382(0.40)$ \\
0.05 & 0.9341 & $0.9477(1.46)$ & $0.9225(1.24)$ & $0.9289(0.56)$ \\
0.07 & 0.9256 & $0.9432(1.90)$ & $0.9109(1.59)$ & $0.9196(0.64)$ \\
0.1 & 0.9114 & $0.9336(2.44)$ & $0.8937(1.94)$ & $0.9060(0.59)$ \\
0.12 & 0.9010 & $0.9252(2.69)$ & $0.8824(2.06)$ & $0.8970(0.45)$ \\
0.14 & 0.8899 & $0.9152(2.84)$ & $0.8713(2.09)$ & $0.8880(0.21)$ \\
0.15 & 0.8840 & $0.9096(2.90)$ & $0.8658(2.06)$ & $0.8836(0.04)$ \\
\hline
\end{tabular}

\section{CONCLUSIONS}

Based on the equivalent linearization method when using the so-called weighted averaging proposed by Anh, a closed-form expression for the optimal turning ratio of a DVA attached to a damped primary structure, modeled as an SDOF system, is presented in this paper. The original damped primary structure is replaced equivalently by an undamped structure and then using the well-known results in case of undamped structures to obtain the analytical expression for the optimal turning ratio. 
The obtained expression of turning ratio is compared with the results in the literature. The comparison shows that the expression of the tuning ratio proposed in this paper coincides with numerical results given by Ioi and Ikeda.

\section{REFERENCES}

[1] H. Frahm. Device for damping vibrations of bodies. U.S. Patent No. 989958, (1909).

[2] J. Ormondroyd and J. Den Hartog. The theory of the dynamic vibration absorber. Transactions of ASME, Journal of Applied Mechanics, 50, (7), (1928), pp. 9-22.

[3] F. Casciati and F. Giuliano. Performance of multi-TMD in the towers of suspension bridges. Journal of Vibration and Control, 15, (6), (2009), pp. 821-847. doi:10.1177/1077546308091455.

[4] N. D. Anh, H. Matsuhisa, L. D. Viet, and M. Yasuda. Vibration control of an inverted pendulum type structure by passive mass-spring-pendulum dynamic vibration absorber. Journal of Sound and Vibration, 307, (1), (2007), pp. 187-201. doi:10.1016/j.jsv.2007.06.060.

[5] P. Warnitchai and N. Hoang. Optimal placement and tuning of multiple tuned mass dampers for suppressing multi-mode structural response. Smart Structures and Systems, 2, (1), (2006), pp. 1-24. doi:10.12989/sss.2006.2.1.001.

[6] J. Den Hartog. Mechanical vibrations. McGraw-Hill, New York, (1956).

[7] T. Ioi and K. Ikeda. On the dynamic vibration damped absorber of the vibration system. Bulletin of the Japanese Society of Mechanical Engineering, 21, (151), (1978), pp. 64-71. doi:10.1299/jsme1958.21.64.

[8] S. E. Randall, D. M. Halsted, and D. L. Taylor. Optimum vibration absorbers for linear damped systems. Journal of Mechanical Design, 103, (4), (1981), pp. 908-913. doi:10.1115/1.3255005.

[9] A. G. Thompson. Optimum tuning and damping of a dynamic vibration absorber applied to a force excited and damped primary system. Journal of Sound and Vibration, 77, (3), (1981), pp. 403-415. doi:10.1016/s0022-460x(81)80176-9.

[10] G. B. Warburton. Optimum absorber parameters for various combinations of response and excitation parameters. Earthquake Engineering \& Structural Dynamics, 10, (3), (1982), pp. 381401. doi:10.1002/eqe.4290100304.

[11] Y. Fujino and M. Abe. Design formulas for tuned mass dampers based on a perturbation technique. Earthquake Engineering \& Structural Dynamics, 22, (10), (1993), pp. 833-854. doi:10.1002/eqe.4290221002.

[12] A. Ghosh and B. Basu. A closed-form optimal tuning criterion for TMD in damped structures. Structural Control and Health Monitoring, 14, (4), (2007), pp. 681-692. doi:10.1002/stc.176.

[13] N. D. Anh and N. X. Nguyen. Extension of equivalent linearization method to design of TMD for linear damped systems. Structural Control and Health Monitoring, 19, (6), (2012), pp. 565573. doi:10.1002/stc.446.

[14] N. D. Anh. Dual approach to averaged values of functions: a form for weighting coefficient. Vietnam Journal of Mechanics, 37, (2), (2015), pp. 145-150. doi:10.15625/0866-7136/37/2/6206.

[15] N. D. Anh, N. Q. Hai, and D. V. Hieu. The equivalent linearization method with a weighted averaging for analyzing of nonlinear vibrating systems. Latin American Journal of Solids and Structures, 14, (9), (2017), pp. 1723-1740. doi:10.1590/1679-78253488. 